\title{
BLOOD COUNTS IN PATIENTS WITH ACUTE HYPERSENSITIVITY REACTIONS TO DRUGS AND FOOD
}

\author{
V. Bulgakova ${ }^{1}$, T. Eliseeva², E. Zastelo², E. Tush², O. Khaletskaya ${ }^{2}$, S. Ignatov ${ }^{3}$, I. Balabolkin ${ }^{1}$ \\ ${ }^{1}$ National Medical Research Center of Children's Health, Institute of Pediatrics, Moscow, Russia. \\ ${ }^{2}$ Privolzhsky Research Medical University, Department of Pediatrics, Nizhny Novgorod, Russia. \\ ${ }^{3}$ Lobachevsky State University of Nizhny Novgorod, Department of Chemistry, Nizhny Novgorod, Russia.
}

\section{Background and Aims}

Adverse drug reactions (ADR) represent a major health problem worldwide with high morbidity and mortality rates. The diagnostic tools available for the practical differentiation of food and drug reactions are very limited and do not allow making a comprehensive picture of the formation of the pathological process in a particular patients.

\section{Method}

Hematological parameters and blood biochemical markers were measured in 134 patients ( 71 boys) aged 4 -17 years with acute hypersensitivity reactions induced by food (65 patients) and medicines (69 patients). Both groups of patients had comparable clinical symptoms with predominance of skin lesions.

\section{Results}

The significant differences between the groups with drug- and food-induced hypersensitivity reactions were found in their red blood characteristics. In patients with hypersensitive reactions to drugs, significantly lower levels of erythrocytes and hemoglobin were found, while the median values of these parameters did not exceed the limits of reference values. These differences persisted also in the analysis of hemoglobin values, analyzed with accounting the age and sex of patients. Reduction of hemoglobin was not accompanied by an increase in bilirubin in these patients.

\section{Conclusion}

Thus, this fact does not support the assumption about the drug-induced hemolysis as a main effect influencing the hematological parameters in these patients. The biochemical markers were not significantly distinguished except bilirubin and alkaline phosphatase which were higher in patients withfood-induced hypersensitivity. The biochemical markers were not significantly distinguished except bilirubin and alkaline phosphatase which were higher in patients withfood-induced hypersensitivity.

\footnotetext{
References

Elzagallaai AA, Greff M, Rieder MJ. Adverse Drug Reactions in Children: The Double-Edged Sword of Therapeutics. Clin Pharmacol Ther. 2017 Jun;101(6):725-735.

Pichler WJ. The area of drug hypersensitivity has been widely neglected not least because it is a complex area. Preface. Med Clin North Am. 2010 Jul;94(4):xv-vi.

Hausmann O1, Schnyder B, Pichler WJ. Etiology and pathogenesis of adverse drug reactions. Chem Immunol Allergy. 2012;97:32-46.
}

Table

Basic hematological values in children with acute food- and drug-induced hypersensitivity reactions

\begin{tabular}{|c|c|c|c|}
\hline \multirow{3}{*}{$\begin{array}{l}\text { Parameters } \\
\text { Number of patients }\end{array}$} & \multicolumn{2}{|c|}{ Group of patients } & \multirow{3}{*}{ Statistics } \\
\hline & Food hypersensitivity & Drug hypersensitivity & \\
\hline & $447+0.38^{63}$ & $421+0.36^{11}$ & \\
\hline & $4.455[4.37 ; 4.5]$ & $4.2[4.1 ; 5.4 .37]$ & $\mathrm{KWT}=9.47 ; p=0.0021$ \\
\hline \multirow[t]{2}{*}{ RBC boys } & $4.53 \pm 0.37$ & $4.30 \pm 0.41$ & $t=5.92 ; p=0.0176$ \\
\hline & $4.46[4.37 ; 4.70]$ & $4.28[4.1 ; 4.5]$ & $K W T=5.19 ; p=0.0233$ \\
\hline RBC girls & $\begin{array}{l}4.38 \pm 0.38 \\
4.39[4.1 ; 4.5]\end{array}$ & $\begin{array}{l}4.24 \pm 0.31 \\
4.2[4.0 ; 4.38]\end{array}$ & $\begin{array}{l}t=2.71 ; p=0.1053 \\
K W T=2.65 ; p=0.104\end{array}$ \\
\hline \multirow[t]{2}{*}{ RRBC } & $1.12 \pm 0.17$ & $1.05 \pm 0.24$ & $t=3.95 ; p=0.0491$ \\
\hline & $1.13[1.11 ; 1.16]$ & $1.08[1.06 ; 1.11]$ & $\mathrm{KWT}=8.47 ; p=0.0036$ \\
\hline \multirow[t]{2}{*}{$H B, g / L$} & $135.64 \pm 11.23$ & $127.20 \pm 8.98$ & $t=23.30 ; p=0.0000$ \\
\hline & $136.0[131.2 ; 142.0]$ & $127.0[124.7 ; 130.3]$ & $K W T=19.27 ; p=0.00001$ \\
\hline \multirow[t]{2}{*}{ HB boys } & $137.78 \pm 11.21$ & $126.15 \pm 10.39$ & $t=20.11 ; p=0.0000$ \\
\hline & $139.0[131.1 ; 143.9]$ & $126.0[120.0 ; 132.3]$ & $K W T=15.37 ; p=0.00009$ \\
\hline \multirow[t]{2}{*}{ HB girls } & $132.6 \pm 10.73$ & $128.1 \pm 7.6$ & $t=3.83 ; p=0.05503$ \\
\hline & $134.5[125.9 ; 142.0]$ & $129.0[124.4 ; 131.6]$ & $K W T=3.91 ; p=0.048$ \\
\hline \multirow[t]{2}{*}{ RHB } & $1.062 \pm 0.084$ & $1.002 \pm 0.072$ & $t=19.95 ; p=0.0000$ \\
\hline & $1.07[1.03 ; 1.11]$ & $1.01[0.98 ; 1.03]$ & $K W T=16.88 ; p=0.00004$ \\
\hline \multirow[t]{2}{*}{ HCT } & $39.4 \pm 2.78$ & $37.60 \pm 2.90$ & $\mathrm{t}=11.87 ; p=0.008$ \\
\hline & $39.85[38.5 ; 41.1]$ & $37.95[36.0 ; 39.0]$ & $K W T=11.26 ; p=0.00008$ \\
\hline \multirow[t]{2}{*}{ HCT boys } & $39.54 \pm 2.52$ & $37.07 \pm 3.24$ & $t=11.38 ; p=0.0013$ \\
\hline & $39.4[38.2 ; 41.3]$ & $36.9[35.1 ; 39.6]$ & $K W T=9.29 ; p=0.002$ \\
\hline \multirow[t]{2}{*}{ HCT girls } & $39.20 \pm 3.17$ & $38.06 \pm 2.51$ & $t=2.25 ; p=0.1397$ \\
\hline & 40.3 [37.96; 41.67] & 38.0 [36.37; 39.83] & $K W T=3.09 ; p=0.079$ \\
\hline \multirow[t]{2}{*}{ MCV } & $81.38 \pm 3.59$ & $80.95 \pm 5.10$ & $t=0.28 ; p=0.5984$ \\
\hline & $80.9[79.9 ; 82.0]$ & 80.751 [78.8; 81.8] & $K W T=0.539 ; p=0.463$ \\
\hline \multirow[t]{2}{*}{ RMCV } & $0.985 \pm 0.041$ & $0.988 \pm 0.051$ & $\mathrm{t}=0.07 ; p=0.7878$ \\
\hline & $0.99[0.98 ; 1.0]$ & $0.985[0.96 ; 1.01]$ & $K W T=0.014 ; p=0.907$ \\
\hline \multirow[t]{2}{*}{ MCH 26-34 } & $27.9 \pm 1.66$ & $27.78 \pm 2.33$ & $\mathrm{t}=0.07 ; p=0.7927$ \\
\hline & 27.7 [27.3; 28.2] & 27.6 [26.8; 28.2] & $K W T=04104 ; p=0.525$ \\
\hline $\mathrm{RMCH}$ & $0.981 \pm 0.054$ & $0.979 \pm 0.075$ & $\mathrm{t}=0.04 ; \mathrm{p}=0.8484$ \\
\hline & $0.98[0.96 ; 0.99]$ & $0.97[0.96 ; 0.99]$ & $K W T=0.414 ; p=0.52$ \\
\hline MCHC 280-370 & $342.72 \pm 15.82$ & $343.5 \pm 22.22$ & $t=0.05 ; p=0.8270$ \\
\hline & $345.0[339.0 ; 350.0]$ & $339.0[335.0 ; 342.2]$ & $\mathrm{KWT}=2.56 ; p=0.11$ \\
\hline $\mathrm{RMCHC}$ & $0.995 \pm 0.046$ & $0.998 \pm 0.064$ & $t=0.11 ; p=0.7364$ \\
\hline & $1.0[0.98 ; 1.01]$ & $0.99[0.97 ; 0.99]$ & $\mathrm{KWT}=1.846 ; p=0.174$ \\
\hline PLT & $293.067 \pm 79.2$ & $300.59 \pm 69.3$ & $t=0.32 ; p=0.5705$ \\
\hline & 290.0 [258.5; 316.2] & $301.0[277 ; 318.5]$ & $K W T=0.5622 ; p=0.4531$ \\
\hline RDW-sd & $38.45 \pm 2.878$ & $39.36 \pm 4.25$ & $\mathrm{t}=1.81 ; p=0.1805$ \\
\hline & $38.0[37.2 ; 39.1]$ & 38.4 [37.7; 39.6] & $K W T=1.167 ; p=0.28$ \\
\hline RDV-cV & $13.35 \pm 0.996$ & $13.54 \pm 0.93$ & $t=1.19 ; p=0.2775$ \\
\hline & $13.1[12.9 ; 13.4]$ & $13.4[13.2 ; 13.77]$ & $\mathrm{KWT}=2.75 ; p=0.1001$ \\
\hline PDW & $12.72 \pm 2.38$ & $11.80 \pm 2.00$ & $t=5.26 ; p=0.0236$ \\
\hline & $12.6[11.8 ; 13.0]$ & $11.4[10.6 ; 12.2]$ & $K W T=5.28 ; p=0.022$ \\
\hline MPV & $10.70 \pm 1.078$ & $10.22 \pm 1.29$ & $\mathrm{t}=4.83 ; \mathrm{p}=0.0299$ \\
\hline & $10.8[10.2 ; 11.0]$ & $10.25[9.6 ; 10.5]$ & $\mathrm{KWT}=4.95 ; \mathrm{p}=0.026$ \\
\hline p-Icr & $30.43 \pm 8.28$ & $27.34 \pm 8.87$ & $t=3.71 ; p=0.0566$ \\
\hline & 30.9 [26.6; 33.0] & $26.8[22.1 ; 29.08]$ & $\begin{array}{l}\mathrm{KWT}=4.1883 ; \mathrm{p}= \\
0.0407\end{array}$ \\
\hline РCT & $0.317 \pm 0.0832$ & $0.318 \pm 0.084$ & $t=0.00 ; p=0.9463$ \\
\hline & $0.31[0.28 ; 0.34]$ & $0.31[0.28 ; 0.33]$ & $\mathrm{KWT}=0.000 ; p=0.996$ \\
\hline WBC & $10.52 \pm 3.80$ & $11.17 \pm 3.94$ & $t=0.92 ; p=0.3394$ \\
\hline & $10.51[8.96 ; 11.38]$ & $10.5[9.43 ; 11.99]$ & $K W T=0.509 ; p=0.476$ \\
\hline NEUT, \% & $77.63 \pm 12.40$ & $73.8 \pm 15.492$ & $t=2.1 ; p=0.1498$ \\
\hline & 79.95 [77.58; 82.94] & $78.2[72.0 ; 82.0]$ & $\mathrm{KWT}=1.43 ; p=0.232$ \\
\hline LYM, \% & $18.28 \pm 10.70$ & $21.28 \pm 12.72$ & $t=1.85 ; p=0.1768$ \\
\hline & $15.58[14.08 ; 19.1]$ & 18.2 [15.28; 22.82] & $\mathrm{KWT}=1.908 ; p=0.167$ \\
\hline MONO, \% & $3.69 \pm 2.42$ & $4.31 \pm 3.58$ & $t=1.15 ; p=0.2857$ \\
\hline & $3.0[2.6 ; 4.17]$ & $3.1[2.2 ; 4.04]$ & $K W T=0.186 ; p=0667$ \\
\hline $\mathrm{EO}, \%$ & $0.34 \pm 0.73$ & $0.627 \pm 1.637$ & $t=1.34 ; p=0.2499$ \\
\hline & $0.1[0.1 ; 0.1]$ & $0.1[0.1 ; 0.2]$ & $K W T=0.601 ; p=0.438$ \\
\hline ESR & $9.33 \pm 6.35$ & $10.06 \pm 5.65$ & $t=0.48 ; p=0.4892$ \\
\hline
\end{tabular}

Abbreviations: WBC - White Blood Cell Count; RBC - Red Blood Cell Count; HGB - Level of hemoglobin molecules; HCT - Hematocrit; MCV - Mean corpuscular volume Red Blood Cell; MCH - Mean corpuscular hemoglobin; MCHC - Mean corpuscular hemoglobin concentration; PLT - Platelets levels in the blood; NEUT\% - Neutrophil granulocyte, \%; MONO\% - Monocyte, \%; EO\% - Eosinophil granulocyte, \%; BASO\% - Basophil granulocyte, \%; RDWSD - red cell distribution width, standard deviation; RDW-CV - red cell distribution width, coefficient of variation; PDW - width of distribution of thrombocytes; MPV - Mean platelet volume; P-LCR - platelet large cell ratio; PCT - platelet crit; volume \% of platelets, ESR - erythrocyte sedimentation rate. 